\title{
Effects on weight gain and gut microbiota in rats given bacterial supplements and a high-energy-dense diet from fetal life through to 6 months of age
}

\author{
Caroline L. J. Karlsson ${ }^{1 *}$, Göran Molin ${ }^{1}$, Frida Fåk ${ }^{2}$, Marie-Louise Johansson Hagslätt ${ }^{3}$, Maja Jakesevic ${ }^{1}$, \\ Åsa Håkansson ${ }^{1}$, Bengt Jeppsson ${ }^{3}$, Björn Weström ${ }^{2}$ and Siv Ahrné ${ }^{1}$ \\ ${ }^{1}$ Food Hygiene, Division of Applied Nutrition and Food Chemistry, Department of Food Technology, Engineering and \\ Nutrition, Lund University, Box 124, SE-221 OO Lund, Sweden \\ ${ }^{2}$ Animal Physiology, Department of Cell and Organism Biology, Lund University, Helgonavägen 3B, SE-223 62 Lund, \\ Sweden \\ ${ }^{3}$ Surgery Research Unit, Department of Clinical Science, Lund University, Skåne University Hospital, SE-205 02 Malmö, \\ Sweden \\ (Received 19 July 2010 - Revised 13 January 2011 - Accepted 27 January 2011 - First published online 30 March 2011)
}

\section{Abstract}

The aim of the present study was to assess the long-term effects of a high-energy-dense diet, supplemented with Lactobacillus plantarum (Lp) or Escherichia coli (Ec), on weight gain, fattening and the gut microbiota in rats. Since the mother's dietary habits can influence offspring physiology, dietary regimens started with the dams at pregnancy and throughout lactation and continued with the offspring for 6 months. The weight gain of group Lp was lower than that of groups C (control) and Ec $(P=0 \cdot 086)$. More retroperitoneal adipose tissue $(P=0.030)$ and higher plasma leptin $(P=0.035)$ were observed in group Ec compared with group Lp. The viable count of Enterobacteriaceae was higher in group Ec than in group Lp $(P=0.019)$, and when all animals were compared, Enterobacteriaceae correlated positively with body weight $(r 0 \cdot 428, P=0.029)$. Bacterial diversity was lower in group Ec than in groups C $(P \leq 0 \cdot 05)$ and Lp $(P \leq 0 \cdot 05)$. Firmicutes, Bacteroidetes and Verrucomicrobia dominated in all groups, but Bacteroidetes were more prevalent in group $\mathrm{C}$ than in groups Lp $(P=0.036)$ and Ec $(P=0.056)$. The same five bacterial families dominated the microbiota of groups Ec and C, and four of these were also present in group Lp. The other five families dominating in group Lp were not found in any of the other groups. Multivariate data analysis pointed in the same directions as the univariate statistics. The present results suggest that supplementation of $L$. plantarum or E. coli can have long-term effects on the composition of the intestinal microbiota, as well as on weight gain and fattening.

Key words: Body weight: Probiotics: Biochemical markers: Gut microbiota: High-energy-dense diet

Although the primary reason for obesity is energy intake exceeding energy expenditure, it is becoming increasingly clear that overweight can also be associated with individual differences in energy extraction capacity, genetic background and gut microbiota $^{(1-6)}$. While intestinal microbes contribute to colonisation resistance, nutrition, and the development and tuning of the immune system ${ }^{(7)}$, studies in both human subjects and animals have also shown a connection between the gut microbiota and obesity ${ }^{(1-5)}$.

Antibiotic treatment is known to manipulate the intestinal microbiota. In experimental studies, it has been postulated that antibiotic treatment during pregnancy impairs the physiology and intestinal microbiota of rat pups ${ }^{(8)}$, and antibiotic modulation of mouse gut microbiota has been shown to change the metabolic status of the host ${ }^{(5)}$.

Since the sterile fetus is colonised by micro-organisms from the mother and the surrounding environment at birth, it can be hypothesised that the microbial heritage from the mother can be important for offspring development and health. Furthermore, the microbiota of the mother can be affected by dietary factors. For example, a high intake of fat can affect the gut microbiota ${ }^{(5)}$.

Constant exposure to bacteria through diet may well have effects in the long run. In the present study, the two non-pathogenic bacteria Lactobacillus plantarum DSM 15313, designated in the division Firmicutes (family Lactobacillaceae), and Escherichia coli CCUG $29300^{\mathrm{T}}$ ( $\mathrm{T}=$ type strain), designated

Abbreviations: C, control; Ec, Escherichia coli CCUG 29300' ; HEDD, high-energy-dense diet; Lp, Lactobacillus plantarum DSM 15313; LPS, lipopolysaccharide; T-RF, terminal restriction fragment; T-RFLP, terminal restriction fragment length polymorphism.

*Corresponding author: C. L. J. Karlsson, fax +4646 2224532, email caroline.karlsson@appliednutrition.lth.se 
in the division Proteobacteria (family Enterobacteriaceae), were given to rat dams and their offspring. L. plantarum frequently occurs spontaneously and in high numbers in most lactic acid-fermented foods, especially those based on plant material, for example, brined olives, capers, sauerkraut, salted gherkins, cassava, and in grape juice and wine ${ }^{(9)}$. However, L. plantarum is also present in the human intestinal mucosa ${ }^{(10)}$, and probiotic effects of certain strains of this species have been documented in human subjects, for example, reduction in abdominal bloating and pain in patients with the irritable bowel syndrome ${ }^{(11)}$ and reduction in CVD risk factors $^{(12)}$. Furthermore, administration of L. plantarum to pregnant dams has been shown to stimulate gut growth and development in their suckling offspring ${ }^{(13)}$. The particular strain, L. plantarum DSM 15313, used in the present study has been shown to mitigate colitis, translocation and liver injury in rats $^{(14,15)}$. Refrigerated foods such as milk, meat and vegetables in an early phase of deterioration, before loss of apparent edibility, expose the consumer to different types of bacteria. Those bacteria are typically Gram-negative Proteobacteria such as Pseudomonas and Enterobacteriaceae that have proinflammatory lipopolysaccharides (LPS) associated with their cell membranes ${ }^{(16)}$. E. coli is a Gram-negative commensal in the gastrointestinal tract. Non-pathogenic strains of $E$. coli can also exert inflammatory stimuli by their LPS, and changed caecal microbiota, as well as increased inflammatory level, have been observed in rat pups with mothers receiving $E$. coli during pregnancy and lactation ${ }^{(8)}$. It has been shown in mice that LPS in the blood (endotoxaemia) is a starting point for insulin resistance and obesity ${ }^{(17)}$. Moreover, it has been depicted that a high-fat diet increased LPS-containing organisms in the gut and increased transfer of LPS into the blood, which resulted in increased systemic inflammation ${ }^{(5)}$.

High biological diversity normally indicates an ecosystem in healthy balance, while a low bacterial diversity in the gastrointestinal tract has been observed in patients with Crohn's disease, ulcerative colitis and atopic eczema ${ }^{(18-20)}$. Recently, obese twins have been reported to have lower intestinal diversity compared with lean twins ${ }^{(4)}$. Interestingly, the probiotic strain L. plantarum DSM 9843 has been shown to improve colonic bacterial diversity in patients with incipient arteriosclerosis $^{(21)}$. However, there is a lack of knowledge of the long-term effect of probiotic administration regarding both gut microbiota and body-weight regulation.

The present study was designed to evaluate the long-term effects on microbiota, weight gain and fattening of regular intake of two fundamentally different non-pathogenic commensal bacteria in combination with a challenging energyrich diet. To further stress the system, by exposure from fetal life to adulthood, rat dams of the outbreed SpragueDawley stock were mated and fed with a high-energy-dense diet (HEDD) and bacterial supplement during pregnancy and lactation, and then their offspring received the same treatment for 6 months.

\section{Experimental methods}

\section{Study design and animals}

For each study group, three adult female Sprague-Dawley rats (Taconic, Ry, Denmark) of similar weight and age were mated with different male rats. From 2 weeks before parturition, dams were housed individually and were assigned diet and bacterial supplements at that time as described below. At weaning, about 4 weeks of age, nine of the female offspring from each study group were randomly mixed into cages with three animals in each cage. They continued in the experiment for 6 months. Throughout the experiment, animals were housed in polycarbonate cages on chopped aspen wood bedding (Beekay bedding; Scanbur BK AB, Sollentuna, Sweden), and the experiment was run in a controlled environment $\left(21 \pm 1^{\circ} \mathrm{C}\right.$ and $50 \pm 10 \%$ relative humidity; $12 \mathrm{~h}$ light $-12 \mathrm{~h}$ dark cycle). The experiment was conducted in compliance with all relevant Swedish laws and institutional guidelines, and the Regional Ethical Review Board, Lund, Sweden, approved the study (approval no. M16-07).

\section{Diets, bacterial supplement and body-weight recordings}

The purified HEDD with $41 \%$ of energy as fat, $41 \%$ of energy as carbohydrates, of which half was sucrose, and $18 \%$ of energy as protein was produced by Research Diets (New Brunswick, NJ, USA; see Table S1 of the supplementary material, available online at http://www.journals.cambridge. org/bjn). Feed was administered ad libitum, and the consumption per cage was recorded once a week. In addition to the HEDD, one group received Lactobacillus plantarum DSM 15313 suspended in freezing medium (4.28 mM$\mathrm{K}_{2} \mathrm{HPO}_{4}, 1.31 \mathrm{~mm}-\mathrm{KH}_{2} \mathrm{PO}_{4}, 1.82 \mathrm{~mm}$-sodium citrate, $0.87 \mathrm{~mm}-$ $\mathrm{MgSO}_{4} \cdot 7 \mathrm{H}_{2} \mathrm{O}$ and $1.48 \mathrm{~mm}$ of $98 \%$ glycerol) (Lp), while another group was given Escherichia coli CCUG $29300^{\mathrm{T}}$ suspended in the freezing medium (Ec) in their drinkingwater. The control (C) group received a corresponding amount of freezing medium in their water. The treatment to dams began during the last two-thirds of pregnancy and continued during lactation. The offspring were then given the same treatment as their mothers until 6 months of age.

A frozen high-density preparation of $L$. plantarum DSM 15313 , originating from the intestine of a healthy human individual, was provided by Probi $\mathrm{AB}$ (Lund, Sweden). E. coli CCUG $29300^{\mathrm{T}}$, a non-pathogenic strain of human origin, was grown in brain heart infusion medium (Oxoid, Basingstoke, Hampshire, UK) in a shaking water-bath at $36^{\circ} \mathrm{C}$ for $16 \mathrm{~h}$. The cells were stored in freezing medium after harvesting. All bacteria were kept at $-80^{\circ} \mathrm{C}$ until feeding. The bacterial suspension was poured into the drinking-water on the day of administration. Water was changed, and consumption was recorded daily. Confirmed by culturing, viable counts of L. plantarum and E. coli were stable during $24 \mathrm{~h}$ in tap water at room temperature. Each cage was given $10^{9}$ colony-forming units/d of L. plantarum or E. coli in $200 \mathrm{ml}$ water. Every rat consumed approximately $10^{8}$ colony-forming units/d of L. plantarum or E. coli. The birth weight of the litter (all pups taken together) was recorded the day after delivery, 
and a mean birth weight of each animal was assigned. Body weight was measured weekly from weaning until killing.

\section{Procedure at animal killing and blood and organ sampling}

At 6 months of age, non-fasted animals were anaesthetised by subcutaneous injection of $0.375 \mathrm{mg}$ fluanisone and $0.012 \mathrm{mg}$ fentanyl citrate/100g body weight (Hypnorm ${ }^{\circledR}$ VetPharma Limited, Leeds, Yorkshire, UK) and $0 \cdot 188 \mathrm{mg}$ midazolam/ $100 \mathrm{~g}$ body weight (Dormicum ${ }^{\circledR}$; Roche, Basel, Switzerland). Under aseptic conditions, a laparotomy was performed through a midline incision. Blood from the aorta was sampled into ice-chilled endotoxin-free polystyrene tubes (Lonza, Copenhagen, Denmark) containing EDTA as an anticoagulant to obtain plasma. Blood was also collected in BD Vacutainer tubes (Becton Dickinson, Plymouth, Devon, UK) to obtain serum. Plasma and serum were stored at $-80^{\circ} \mathrm{C}$ until further analyses. Plasma for the determination of endotoxin was stored in endotoxin-free tubes (Biopur $1.5 \mathrm{ml}$ tubes; Eppendorf, Hamburg, Germany). Left retroperitoneal and periovarial adipose tissue, spleen and adrenals were carefully dissected and weighed. Caecal and rectal content and liver tissue were frozen, in freezing medium for culturing, in liquid $\mathrm{N}_{2}$, and stored at $-80^{\circ} \mathrm{C}$ until analyses.

\section{Biochemical analyses}

Plasma leptin was analysed using ELISA (Crystal Chem, Inc., Downers Grove, IL, USA), with the detection limit of $0 \cdot 2 \mathrm{ng} / \mathrm{ml}$. Plasma haptoglobin was analysed colorimetrically, with an assay sensitivity of $0.05 \mathrm{mg} / \mathrm{ml}$ (Phase ${ }^{\mathrm{TM}}$ Range Haptoglobin Assay; Tridelta Development Limited, Kildare, Republic of Ireland), according to the manufacturer's instructions. Plasma endotoxin was determined by an accredited chromogenic endpoint method based on limulus amebocyte extract (Charles River Laboratories, Charleston, SC, USA). Samples were diluted $1: 10$ and heated at $75^{\circ} \mathrm{C}$ for $10 \mathrm{~min}$; the detection limit was $0.03 \mathrm{IU} / \mathrm{ml}$. Each sample was spiked with endotoxin, and $50 \%$ recovery was ensured in order to analyse lack of inhibitors and verify reliability. Serum bilirubin, aspartate aminotransferase, alanine aminotransferase and alkaline phosphatase were analysed colorimetrically, and rectal content of calprotectin was analysed using ELISA (Immundiagnostic AG, Bensheim, Germany).

\section{Liver histology}

Liver tissue was fixed in $4 \%$ buffered formaldehyde (Histolab, Göteborg, Sweden), rinsed in water and dehydrated in graded ethanol concentrations, followed by xylene and embedded in paraffin. Sections $(5 \mu \mathrm{m})$ were mounted on slides, deparaffinised and stained with haematoxylin and eosin, and the prevalence of steatosis was scored as follows: 0 , no visible steatosis; 1 , steatosis in $<1 / 3$ of the hepatocytes; 2 , steatosis in $1 / 3-2 / 3$ of the hepatocytes; 3 , steatosis in $>2 / 3$ of the hepatocytes. The histological evaluation was performed by a blinded scientist.

\section{Microbial analyses}

In order to analyse the amount of viable lactobacilli and Enterobacteriaceae in the caecum, a conventional dilution series was performed, and samples from appropriate dilutions were plated on Rogosa agar (Oxoid) and violet red bile dextrose agar (Oxoid) and incubated at $37^{\circ} \mathrm{C}$ for $72 \mathrm{~h}$ anaerobically and $24 \mathrm{~h}$ aerobically, respectively. Translocation of viable bacteria was evaluated by anaerobic and aerobic cultivation of liver tissue on the brain heart infusion agar (Oxoid) at $37^{\circ} \mathrm{C}$ for $72 \mathrm{~h}$. Microbial diversity was assessed by the terminal restriction fragment length polymorphism (T-RFLP) method, and cloning and sequencing were used to imply phylogenetic affiliations of the intestinal microbiota. The procedures are described in the supplementary material (available online at http://www.journals.cambridge.org/bjn).

\section{Calculations}

The relative abundance of each terminal restriction fragment (T-RF) within a given T-RFLP pattern was calculated as the peak area of the respective T-RF divided by the total peak area of all T-RF, in the given T-RFLP pattern, detected within a fragment length of 20-600 bp. Relative abundance was used for calculations of diversity index. Shannon and Weaner (Shannon, $H^{\prime}$ ) and Simpson $(D)$ indices were calculated for all individuals by using the equations:

$$
H^{\prime}=-\sum p_{i} \ln p_{i} \text { and } 1-D,
$$

where $D=\Sigma p_{i}^{2}$ and $p_{i}$ is the relative abundance of the $i$ th peak in the community ${ }^{(22)}$. T-RF incidences were calculated using Fisher's exact test, and two-tailed $P$ values were reported.

Statistical evaluation was performed using the KruskalWallis test followed by the Nemenyi-Damico-Wolfe-Dunn test $^{(23)}$ using the package coin ${ }^{(24)}$ in the statistical software $\mathrm{R}$ 2.9.1 (The R Foundation for Statistical Computing, Vienna, Austria). Spearman rank order correlation coefficients were calculated using SigmaStat 3.1 (Systat Software, Point Richmond, CA, USA), and Fisher's exact test was performed with Statistics Online Computational Resource (http://www. socr.ucla.edu/SOCR.html). A $P$ value of $<0.05$ was considered significant.

Multivariate data analysis with orthogonal partial least squares to latent structures discriminant analysis was performed using SIMCA-P + 12.0.1 (Umetrics, Umeå, Sweden) to reveal differences between the treatment groups ${ }^{(25,26)}$.

\section{Results}

\section{Animal health and performance}

All animals (nine animals per group) appeared healthy throughout the study, with the exception of one rat in group Ec that was killed after 12 weeks after veterinarian consultation and recommendation, due to the finding of a subcutaneous tumour. Data from this rat were excluded. Of the remaining Ec rats, one was perceptibly leaner than the others, as confirmed at dissection at the end of the study. 


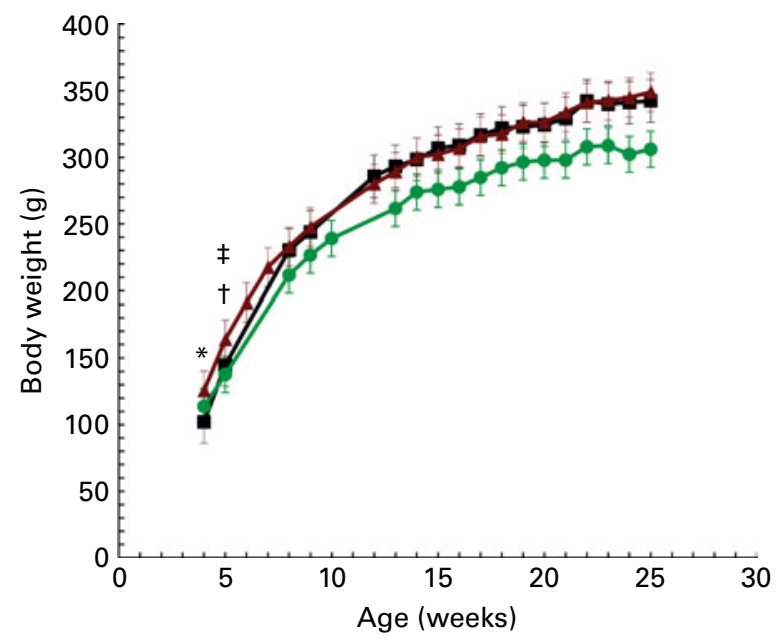

Fig. 1. Body weight development. Values are means (from weaning until 6 months of age), with their standard errors represented by vertical bars. * The body weights were significantly different at 4 weeks; the group exposed to Escherichia coli CCUG $29300^{\top}$ (Ec, A) had a significantly higher body weight than the control group $(C)(P=0.002)$. The body weights were significantly different at 5 weeks; group Ec was heavier than groups $C(\square)$ and the group exposed to Lactobacillus plantarum DSM 15313 ( $\mathrm{Lp}, \bullet$ ): $† P=0.003$; $\ddagger P=<0.001$, respectively. The body weights were not significantly different after 6 months, but group Lp tended to have a significantly lower body weight $(P=0.086)$.

No signs of disease could be diagnosed, and this rat was included in the data evaluation. No difference in feed and water consumption was observed among the groups.

\section{Effects on body and organ weights}

Pups in group Lp had the lowest $(7.47 \mathrm{~g})$ and group Ec had the highest $(9.65 \mathrm{~g})$ mean birth weight, while group $\mathrm{C}$ had intermediate birth weight ( $8 \cdot 15 \mathrm{~g})$. However, because of the limited number of litters, statistical analysis could not be performed. Group Lp had lower body weight throughout the study compared with groups $\mathrm{C}$ and Ec, and at 6 months; the difference approached statistical significance $(P=0.086$; Fig. 1 and Table 1). Significantly more retroperitoneal fat was observed in group Ec compared with group Lp $(P=0.03$; Table 1$)$. The weight of retroperitoneal fat correlated positively with body weight after 6 months $(r 0.454, P=0.02)$. Group Ec had a higher median weight of the periovarial fat deposit compared with the other groups, but the difference was not significant (Table 1). The weight of spleen was higher in group Ec than in group Lp $(P=0 \cdot 006$; Table 1$)$. Group Lp had heavier adrenals than group $\mathrm{C}(P=0 \cdot 022$; Table 1$)$.

\section{Effects on liver histology}

The incidence of liver steatosis was significantly lower in group Ec compared with that in group $\mathrm{C}(P=0 \cdot 008$; Table 1$)$.

\section{Effects on biochemical markers}

Group Ec showed significantly higher plasma leptin concentrations than group Lp $(P=0.035$; Table 2$)$. Haptoglobin was significantly lower in group Ec compared with group $\mathrm{C}$ ( $P=0.003$; Table 2), but no significant differences were observed in the blood levels of endotoxin, bilirubin or the liver enzymes aspartate aminotransferase, alanine aminotransferase and alkaline phosphatase, nor in intestinal calprotectin (Table 2). However, group $\mathrm{C}$ had the highest aspartate aminotransferase concentration, which approached statistical significance $(P=0 \cdot 087)$.

\section{Effects on microbiota}

Significantly lower bacterial diversity was observed in the caecum of group Ec compared with that in group $\mathrm{C}$ when diversity indices were calculated for the T-RFLP profiles (Table 3). When MspI was used for DNA digestion, group Ec had significantly lower diversity than group Lp (Table 3).

Clone libraries from the different groups showed that divisions Firmicutes, Bacteroidetes and Verrucomicrobia were represented in all three groups at a level ranging 65-77, 4-26 and 5-15\%, respectively. At the hierarchical level of family, the same five taxa dominated the microbiota in animals with the most diverse microbiota (according to T-RFLP profiles) in groups $\mathrm{C}$ and $\mathrm{Ec}$, while nine taxa were seen in group Lp (Fig. 2). As regards proportions of the microbiota, significant differences were observed between the treatment groups at division level. Members of the Bacteroidetes were less prevalent in the Ec library than in the C library (LibCompare in Ribosomal Database Project, $P=0.036$ ) and

Table 1. Body weights, weights of fat deposits, spleen weight, adrenal weights and liver steatosis in rats fed a high-energy-dense diet with bacterial supplements for 6 months

(Medians and interquartile ranges)

\begin{tabular}{|c|c|c|c|c|c|c|}
\hline & \multicolumn{2}{|c|}{ Group C ( $n 9)$} & \multicolumn{2}{|c|}{ Group Lp ( $n 9)$} & \multicolumn{2}{|c|}{ Group Ec ( $n 8)$} \\
\hline & Medians & Interquartile ranges & Medians & Interquartile ranges & Medians & Interquartile ranges \\
\hline Body weight $(\mathrm{g})$ & $340 \cdot 6^{a}$ & $313 \cdot 8-373 \cdot 4$ & $303.8^{\mathrm{a}}$ & $298 \cdot 3-306 \cdot 6$ & $351 \cdot 8^{a}$ & $300 \cdot 1-401 \cdot 7$ \\
\hline Left retroperitoneal fat deposit $(\mathrm{mg})$ & $1674^{\mathrm{a}, \mathrm{b}}$ & $1547-1824$ & $1308^{\mathrm{a}}$ & $1103-1496$ & $2903^{b}$ & $1532-4115$ \\
\hline Left periovarial fat deposit $(\mathrm{mg})$ & $5777^{\mathrm{a}}$ & $5040-5969$ & $4689^{a}$ & $4584-5161$ & $8548^{a}$ & $4451-12152$ \\
\hline Spleen $(\mathrm{mg})$ & $674^{\mathrm{a}, \mathrm{b}}$ & $564-700$ & $620^{\mathrm{a}}$ & $598-626$ & $735^{\mathrm{b}}$ & $680-839$ \\
\hline Adrenals (mg) & $61^{a}$ & $60-63$ & $70^{\mathrm{b}}$ & $65-72$ & $65^{\mathrm{a}, \mathrm{b}}$ & $64-72$ \\
\hline Liver steatosis scoring & $2^{\mathrm{a}}$ & $1-2$ & $1^{\mathrm{a}, \mathrm{b}}$ & $1-1$ & $0^{\mathrm{b}}$ & $0-1$ \\
\hline
\end{tabular}

C, control; Lp, Lactobacillus plantarum DSM 15313; Ec, Escherichia coli CCUG $29300^{\top}$.

${ }_{\mathrm{a}, \mathrm{b}}$ Values within a row with unlike superscript letters were significantly different $(P \leq 0.05)$. 
Table 2. Concentrations of biochemical markers in rats fed a high-energy-dense diet with bacterial supplements for 6 months (Medians and interquartile ranges)

\begin{tabular}{|c|c|c|c|c|c|c|}
\hline & \multicolumn{2}{|c|}{ Group C ( $n 9)$} & \multicolumn{2}{|c|}{ Group Lp (n 9) } & \multicolumn{2}{|c|}{ Group Ec ( $n$ 8) } \\
\hline & Median & Interquartile range & Median & Interquartile range & Median & Interquartile range \\
\hline Leptin in blood $(\mathrm{ng} / \mathrm{ml})$ & $6 \cdot 20^{a, b}$ & $5 \cdot 24-7 \cdot 60$ & $4.47^{\mathrm{a}}$ & $3.73-5.75$ & $9.89^{b}$ & $7.04-12.53$ \\
\hline Haptoglobin in blood $(\mathrm{mg} / \mathrm{ml})$ & $0.58^{\mathrm{a}}$ & $0.43-0.82$ & $0.35^{a, b}$ & $0.33-0.49$ & $0.22^{\mathrm{b}}$ & $0.10-0.38$ \\
\hline Endotoxin in blood (IU/ml) & $0.031^{\mathrm{a}}$ & $<0.030-0.035$ & $<0.030^{\mathrm{a}}$ & $<0.030-0.036$ & $0.035^{\mathrm{a}}$ & $0.033-0.045$ \\
\hline Bilirubin in blood $(\mu \mathrm{mol} / \mathrm{l})$ & $2 \cdot 0^{\mathrm{a}}$ & $1 \cdot 8-2 \cdot 2$ & $1.6^{\mathrm{a}}$ & $1.5-1.7$ & $1.9^{\mathrm{a}}$ & $1.8-1.95$ \\
\hline AST in blood $(\mu \mathrm{kat} / /)^{*}$ & $1.80^{\mathrm{a}}$ & $1.74-1.95$ & $1.33^{\mathrm{a}}$ & $1.21-1.54$ & $1.27^{\mathrm{a}}$ & $1.26-1.30$ \\
\hline ALT in blood $(\mu \mathrm{kat} / \mathrm{l})$ & $0.49^{\mathrm{a}}$ & $0.41-0.54$ & $0.40^{\mathrm{a}}$ & $0.34-0.47$ & $0.44^{\mathrm{a}}$ & $0.39-0.46$ \\
\hline ALP in blood $(\mu \mathrm{kat} / \mathrm{l})$ & $2 \cdot 0^{\mathrm{a}}$ & $1 \cdot 7-2 \cdot 1$ & $1 \cdot 7^{\mathrm{a}}$ & $1.7-1.9$ & $1.5^{\mathrm{a}}$ & $1 \cdot 3-2 \cdot 1$ \\
\hline Calprotectin in the intestine $(\mathrm{ng} / \mathrm{ml})$ & $1.91^{a}$ & $1.76-1.99$ & $1.96^{\mathrm{a}}$ & $1.92-2.02$ & $1.89^{a}$ & $1.84-1.94$ \\
\hline
\end{tabular}

C, control; Lp, Lactobacillus plantarum DSM 15313; Ec, Escherichia coli CCUG $29300^{\top}$; AST, aspartate aminotransferase; ALT, alanine aminotransferase; ALP, alkaline phosphatase.

a,b Median values within a row with unlike superscript letters were significantly different $(P \leq 0.05)$

${ }^{*} P=0.087$.

were close to significance for less prevalence in the Lp library compared with the C library $(P=0.056)$. No significance in proportions of sequences belonging to Firmicutes was observed. All sequences of Verrucomicrobia, independent of the treatment group, corresponded to Akkermansia muciniphila. Incertae sedis XIII included sequences most similar to those of Anaerovorax odorimutans but with a low similarity score (0.94). Incidence of T-RF differed significantly between groups Lp and $\mathrm{C}$, and between groups $\mathrm{Ec}$ and $\mathrm{C}$ (see Table S2 of the supplementary material, available online at http://www.journals.cambridge.org/bjn). The gut microbiota was also significantly different in groups Lp and Ec, since group Ec, with $A l u \mathrm{I}$ digestion, had the highest incidence of T-RF at $77 \mathrm{bp}$ and the lowest incidence of $203 \mathrm{bp}$. With Msp I digestion, the T-RF at $257 \mathrm{bp}$ was higher, and T-RF of 80, 258 and $516 \mathrm{bp}$ was found at significantly lower incidence in group Ec than in group Lp.

The viable count of Enterobacteriaceae in the caecal content was significantly higher in group Ec compared with that in group Lp ( $P=0.019$; Table 3$)$ and correlated positively with body weight after 6 months $(r$ 0.428, $P=0.029)$. No significant differences were observed in the lactobacilli count of caecal content or the translocation of viable bacteria to the liver.

\section{Multivariate analysis}

Results of multivariate data analysis with orthogonal partial least squares to latent structure discriminant analysis depict a substantial difference between the different groups. Important for the separation of groups Lp and Ec were fat deposition, viable count of Enterobacteriaceae and microbial diversity (Fig. 3). Significant for the separation of groups Ec and $\mathrm{C}$ were fat deposition and diversity indices, while separation of groups Lp and $\mathrm{C}$ were based on body weight and weight of adrenals, Q2 0.79 and 0.58, respectively.

\section{Discussion}

Feeding with an HEDD and the two bacterial supplements was initiated during pregnancy, because it is known that metabolic malprogramming in the offspring can occur when the dietary intake of the pregnant dam is manipulated ${ }^{(27,28)}$. In the present study, E. coli was chosen as one supplement, since it has been shown that this Gram-negative bacterium, with pro-inflammatory LPS associated with its cell wall, can occasionally dominate the intestinal microbiota in human infants $^{(29)}$. E. coli was also observed to dominate in the sigmoidal mucosa in one out of nine 60-year-old volunteers

Table 3. Microbial diversity in caecal content, calculated with Shannon and Simpson diversity indices, and viable counts of Enterobacteriaceae and lactobacilli

(Medians and interquartile ranges)

\begin{tabular}{|c|c|c|c|c|c|c|}
\hline & \multicolumn{2}{|c|}{ Group C ( $n 9)$} & \multicolumn{2}{|c|}{ Group Lp $(n 9)$} & \multicolumn{2}{|c|}{ Group Ec ( $n 8)$} \\
\hline & Median & Interquartile range & Median & Interquartile range & Median & Interquartile range \\
\hline \multicolumn{7}{|l|}{ Diversity index, restriction endonuclease } \\
\hline Shannon, Alul & $2 \cdot 85^{a}$ & $2 \cdot 69-2 \cdot 89$ & $2 \cdot 74^{\mathrm{a}, \mathrm{b}}$ & $2 \cdot 66-2 \cdot 90$ & $2 \cdot 50^{\mathrm{b}}$ & $2 \cdot 32-2 \cdot 63$ \\
\hline Shannon, Mspl & $2 \cdot 87^{a}$ & $2.65-2.92$ & $2 \cdot 82^{\mathrm{a}}$ & $2 \cdot 78-2.95$ & $2 \cdot 48^{\mathrm{b}}$ & $2 \cdot 23-2 \cdot 60$ \\
\hline Simpson, Alul & $0.91^{\mathrm{a}}$ & $0.89-0.92$ & $0.89^{a, b}$ & $0.86-0.91$ & $0.86^{\mathrm{b}}$ & $0.82-0.88$ \\
\hline Simpson, Mspl & $0.91^{\mathrm{a}}$ & $0.90-0.93$ & $0.90^{\mathrm{a}}$ & $0.84-0.93$ & $0.88^{\mathrm{a}}$ & $0.83-0.93$ \\
\hline \multicolumn{7}{|l|}{ Viable count } \\
\hline Enterobacteriaceae (log cfu/g caecal content) & $5 \cdot 57^{\mathrm{a}, \mathrm{b}}$ & $5 \cdot 28-6 \cdot 29$ & $4.91^{\mathrm{a}}$ & $4 \cdot 63-5 \cdot 13$ & $5 \cdot 94^{\mathrm{b}}$ & $5 \cdot 50-6 \cdot 63$ \\
\hline Lactobacilli (log cfu/g caecal content) & $6.57^{\mathrm{a}}$ & $6.50-7.38$ & $7 \cdot 15^{\mathrm{a}}$ & $6.98-7.74$ & $6 \cdot 56^{\mathrm{a}}$ & $6 \cdot 37-6 \cdot 72$ \\
\hline
\end{tabular}

C, control; Lp, Lactobacillus plantarum DSM 15313; Ec, Escherichia coli CCUG $29300^{\top}$; cfu, colony-forming units.

a,b Median values within a row with unlike superscript letters were significantly different $(P \leq 0.05)$. 


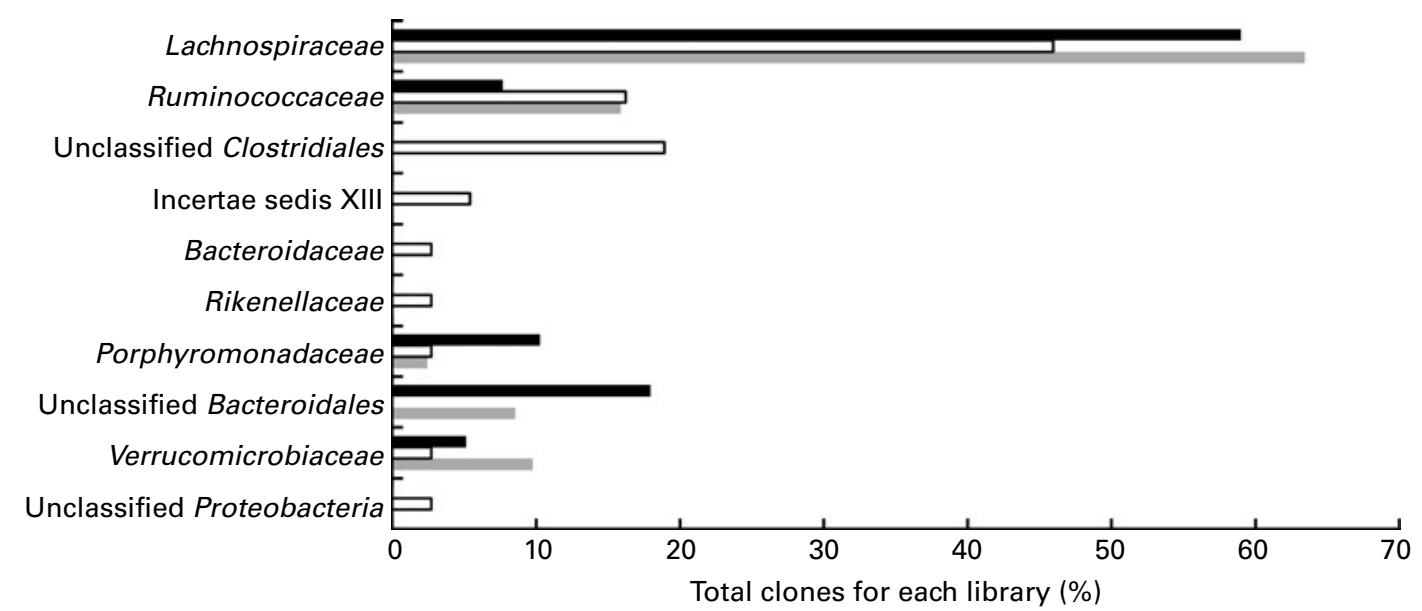

Fig. 2. Identification of sequences in clone libraries to the level of family. The figure shows the families to which clones in each library are related. Results are given as percentage of total number of clones for each specific library. In the control group ( $\square$ ), forty-one clones were sequenced, and in the Lactobacillus plantarum DSM $15313(\square)$ and Escherichia coli CCUG 29300 $(\square)$ groups, forty-four and eighty-four clones were sequenced, respectively.

without clinical symptoms or medication when sequence analysis of cloned $16 \mathrm{~S}$ rRNA genes, as enriched by PCR from biopsies, was applied (91\% of the clone library were related to E. coli; G Molin, unpublished results). In contrast, L. plantarum was selected since it is a Gram-positive commensal of the gastrointestinal tract and can confer health benefits as probiotics ${ }^{(10,11)}$

In the present 6-month study, long-term bacterial supplementation seemed to affect the weight development of the rats, without having any effect on the feed consumption. Furthermore, when repeated-measures ANOVA was calculated, group Lp had significantly lower body weight compared with group C (data not shown). Importantly, L. plantarum decreased body-weight gain, and E. coli increased body fat, the latter being consistent with the hypothesis of Cani et $a l{ }^{(5)}$, which suggests increased body-weight gain by highfat feeding and an increased load of Gram-negative bacteria. To our knowledge, effects of probiotics on weight gain have not previously been experimentally evaluated from fetal life to adulthood. However, Lactobacillus gasseri SBT2055 has been shown to significantly reduce abdominal visceral and subcutaneous fat ${ }^{(30)}$, and lower BMI has been suggested in children after Lactobacillus rhamnosus GG administration ${ }^{(31)}$.

Intestinal diversity has previously been shown to increase in rodents receiving probiotics ${ }^{(32,33)}$. However, these findings were from short-term studies, in which no physiological parameters were evaluated. Thus, there is a general lack of knowledge regarding the long-term effects of bacterial supplements on intestinal microbial diversity in combination with changed homeostasis. Calculation of diversity index is a common way to estimate microbial diversity ${ }^{(18,20-22)}$, and low diversity has been associated with various diseases ${ }^{(18-20)}$.

In the present study, reduced bacterial diversity indices were observed in group Ec compared with group C. Group Ec had a significantly increased viable count of Enterobacteriaceae in the caecum compared with group Lp, as well as lower diversity indices, thereby indicating that $E$. coli consumption might associate with a tendency of overgrowth of unfavourable bacterial groups. Furthermore, the amount of Enterobacteriaceae in the caecal content was positively correlated with body weight. The incidence of specific T-RF varied among the groups, which further supports the observation of different intestinal microbiota according to treatment.

Previous reports have suggested a reduction in the abundance of Bacteroidetes and a proportional increase in Firmicutes in obese individuals ${ }^{(2,3)}$. However, this was not the case in the present study as both the groups of the heavier Ec animals and the leaner Lp animals had lower proportions of Bacteroidetes compared with group C. Furthermore, the proportion of Firmicutes did not vary between the groups. This suggests that the microbiota must be dealt with on lower hierarchical levels than that of division, preferably on the species level but at least at family and genus levels. It also seems important to look into different experimental models with different strains and species of animals, and also different categories of human subjects. Hence, more extensive studies are required to fully explain the connection between gut microbiota and obesity.

In an attempt to evaluate the correlation of intestinal microbiota and changed homeostasis, we measured various biochemical markers. Previously, adult offspring to LPSexposed dams have been reported to have higher body weight, heavier fat deposits and higher serum leptin ${ }^{(34)}$. The same scenario was true in the present study for the group where the mothers and the offspring received E. coli. Interestingly, the opposite effect was observed in the group receiving L. plantarum: lower leptin levels were observed in combination with lower body weight.

The acute-phase protein haptoglobin, mainly produced by the liver ${ }^{(35)}$, is known to increase during inflammation, but contradictory results have also been reported ${ }^{(36,37)}$. Since obesity is characterised by low-grade inflammation ${ }^{(5,38)}$ and group Ec in the present study had more body fat and higher leptin levels, the animals in this group were assumed to have a higher inflammatory tone. In spite of this, group Ec had significantly lower haptoglobin concentrations compared with group C. Interestingly, one of the primary 

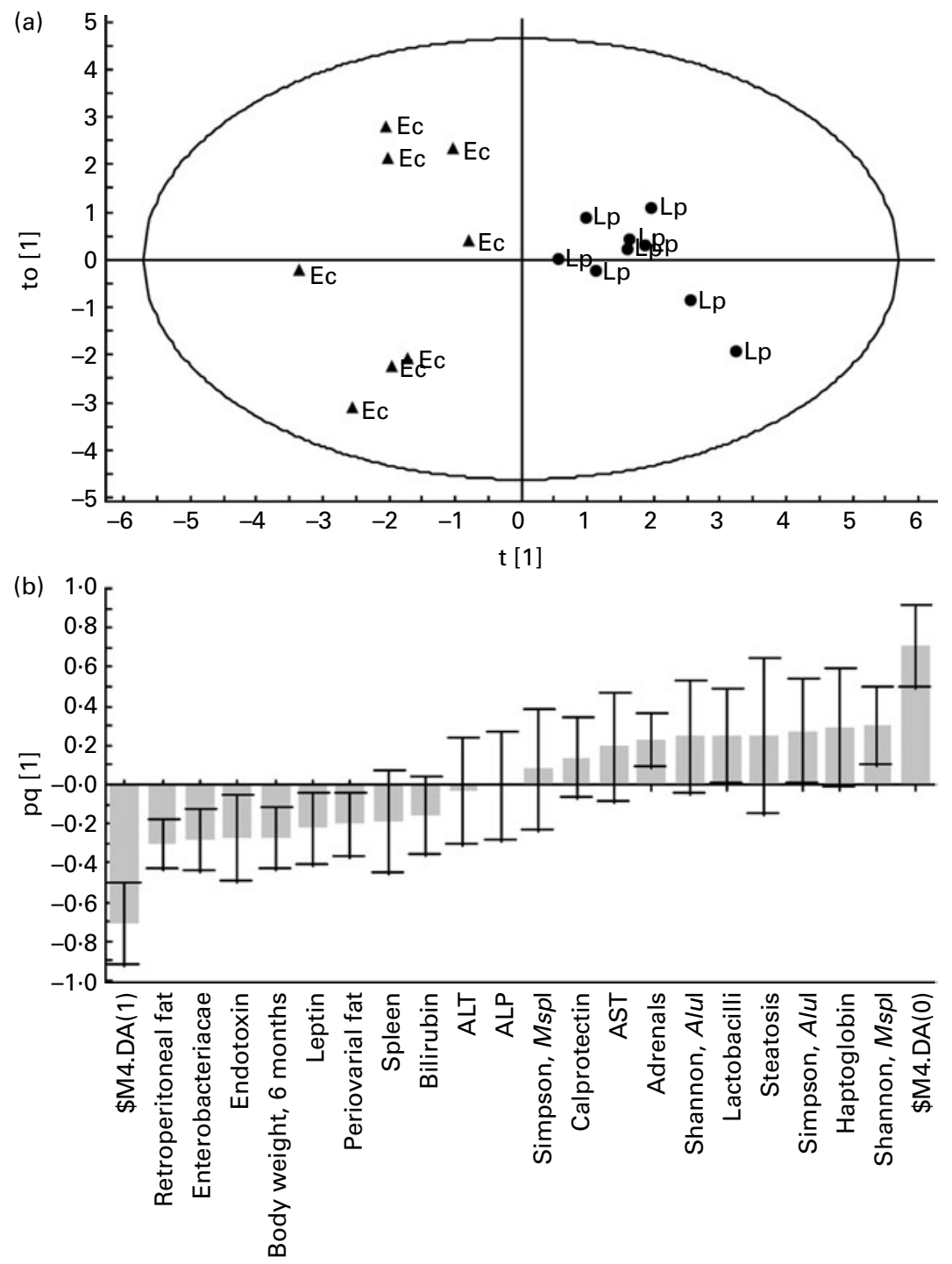

Var ID (primary)

Fig. 3. Multivariate analysis with orthogonal partial least squares to latent structures (OPLS-DA). OPLS-DA score and loading column plots ((a) and (b), respectively) to discriminate the correlation between the Lactobacillus plantarum DSM 15313 (Lp) and Escherichia coli CCUG 29300 ${ }^{\top}$ (Ec) groups. Fat deposition, viable count of Enterobacteriaceae and diversity of the intestinal microbiota were important for the separation of groups Lp and Ec. Q2 was 0.55. ALT, alanine aminotransferase; ALP, alkaline phosphatase; AST, aspartate aminotransferase; $\bullet, 0 ; \mathbf{\Lambda}, 1 ; \mathrm{t}$ [1], 1st score vector; to [1], orthogonal 1st vector; pq [1], 1st predicted OPLS component ( $p$ loading for $x$ and $q$ loading for $y$ ); Var ID, variable ID.

functions of haptoglobin is to restrict $\mathrm{Fe}$ availability to pathogenic bacteria by the formation of an irreversible complex with $\mathrm{Hb}$ released from damaged erythrocytes ${ }^{(39,40)}$. The decreased haptoglobin concentration found in group Ec might be a result of even non-pathogenic E. coli strains being equipped with efficient means (siderophores) to absorb $\mathrm{Fe}^{(41)}$. In the present study, significantly heavier spleens were found in group Ec compared with group Lp, indicating systemic inflammation in group $\mathrm{Ec}^{(42)}$. Furthermore, intravascular haemolysis is associated with splenomegaly and decreased haptoglobin levels ${ }^{(43)}$.

Esposito et $a l^{(44)}$ reported reduced liver damage and inflammation in young rats on a high-fat diet when this was combined with the probiotic mixture VSL $\# 3$ containing different strains of Lactobacillus and Bifidobacterium, but no microbial evaluation was performed. In the present study, no statistically significant changes in liver enzymes were observed. However, aspartate aminotransferase and haptoglobin concentrations were highest in group $\mathrm{C}$, which is in accordance with the higher occurrence of liver steatosis in this group. Non-pathogenic strains of E. coli have indicated improvement of liver function, while other Enterobacteriaceae showed the opposite ${ }^{(45)}$. This highlights the potential to influence the level of steatosis and liver function by modulating the intestinal microbiota. Notably, in the present study, eating behaviour was not controlled before blood sampling at killing, and, for example, plasma LPS is enhanced after a meal ${ }^{(46)}$, thus some measurements may be on the small side.

It should be stressed that in the present study, calprotectin in the intestinal content did not vary between the 
groups, suggesting absence of intestinal inflammation. Hence, although systemic effects were observed, neither $E$. coli nor L. plantarum seemed to have injured the gastrointestinal tract.

The weight of adrenals was significantly higher in group Lp than in group $\mathrm{C}$, but it was not possible to distinguish the weights of the medulla and cortex, making it hard to evaluate the consequences and underlying relevance of the difference in the weight of this organ.

Although not totally conclusive, the present results indicate that it may be possible to affect host homeostasis by bacterial supplementation to rats consuming an HEDD. In future studies, larger study populations should be used, and physiological and microbial characterisation of rat dams should be addressed more extensively. Furthermore, extensive evaluation of the offspring early in life should be performed to distinguish the effect obtained on the fetus in utero, during the neonatal and weaning periods. Litter sizes and social behaviour, such as cage hierarchy, should also be considered.

In summary, the long-term effects of bacterial supplements to HEDD-fed rats were studied from fetal life to adulthood in outbreed rats. Animals receiving L. plantarum had lower body weight, less retroperitoneal adipose tissue and lower plasma leptin compared with those that were given $E$. coli. The diversity of the intestinal microbiota was reduced in rats consuming E. coli, which highlights the potential of modulating intestinal microbiota and homeostasis. As shown by the multivariate analysis, fat deposition and weight gain were affected by the gut microbiota, and manipulated by the supplementation of bacteria to an HEDD.

\section{Acknowledgements}

Mrs Camilla Björklöv is thanked for her assistance with the animal care and Mrs Susanne Eiswold for embedding of the liver tissues. Dr Fredrik Nilsson at the Competence Centre for Clinical Research, Region Skåne (Lund, Sweden), is greatly acknowledged for his statistical recommendations and calculations in R, and Martin Berntsson at Umetrics (Umeå, Sweden), for his guidance in multivariate evaluation of the results. The present study was supported by grant from the Swedish Research Council Formas (grant no. 222-2007-501). G. M., B. J. and S. A. are minority shareholders in Probi AB (Lund, Sweden), and the authors' relationship between the company and their positions at the university has been regulated by an agreement between Lund University (Lund, Sweden) and Probi AB (Lund, Sweden). C. L. J. K., F. F., M.-L. J. H., M. J., Å. H. and B. W. disclose no conflicts of interest. C. L. J. K., G. M., F. F., M. J., A. H., B. J., B. W. and S. A. designed the study; C. L. J. K., F. F., M. J. and A. H. collected the data; C. L. J. K., G. M., M.-L. J. H. and S. A. analysed and interpreted the data; C. L. J. K., G. M. and S. A. wrote the manuscript; all authors read and approved the final version of the manuscript.

\section{References}

1. Bäckhed F, Ding H, Wang T, et al. (2004) The gut microbiota as an environmental factor that regulates fat storage. Proc Natl Acad Sci U S A 101, 15718-15723.
2. Ley RE, Bäckhed F, Turnbaugh P, et al. (2005) Obesity alters gut microbial ecology. Proc Natl Acad Sci U S A 102, 11070-11075.

3. Ley RE, Turnbaugh PJ, Klein S, et al. (2006) Microbial ecology: human gut microbes associated with obesity. Nature 444, 1022-1023.

4. Turnbaugh PJ, Hamady M, Yatsunenko T, et al. (2009) A core gut microbiome in obese and lean twins. Nature $\mathbf{4 5 7}$, 480-484.

5. Cani PD, Bibiloni R, Knauf C, et al. (2008) Changes in gut microbiota control metabolic endotoxemia-induced inflammation in high-fat diet-induced obesity and diabetes in mice. Diabetes 57, 1470-1481.

6. Turnbaugh PJ, Ley RE, Mahowald MA, et al. (2006) An obesity-associated gut microbiome with increased capacity for energy harvest. Nature 444, 1027-1031.

7. McFarland LV (2000) Normal flora: diversity and functions. Microb Ecol Health Dis 12, 193-207.

8. Fåk F, Ahrné S, Molin G, et al. (2008) Microbial manipulation of the rat dam changes bacterial colonization and alters properties of the gut in her offspring. Am J Physiol Gastrointest Liver Physiol 294, G148-G154.

9. Molin G (2008) Handbook of Fermented Functional Foods, 2nd ed. [G Mazza, editor]. Boca Raton, FL: CRC Press \& Taylor \& Francis Group.

10. Molin G, Jeppsson B, Johansson ML, et al. (1993) Numerical taxonomy of Lactobacillus spp. associated with healthy and diseased mucosa of the human intestines. I Appl Bacteriol 74, 314-323.

11. Nobaek S, Johansson ML, Molin G, et al. (2000) Alteration of intestinal microflora is associated with reduction in abdominal bloating and pain in patients with irritable bowel syndrome. Am J Gastroenterol 95, 1231-1238.

12. Naruszewicz M, Johansson ML, Zapolska-Downar D, et al. (2002) Effect of Lactobacillus plantarum 299v on cardiovascular disease risk factors in smokers. Am J Clin Nutr 76, 1249-1255.

13. Fåk F, Ahrné S, Molin G, et al. (2008) Maternal consumption of Lactobacillus plantarum 299v affects gastrointestinal growth and function in the suckling rat. BrJ Nutr 100, 332-338.

14. Osman N, Adawi D, Ahrné S, et al. (2007) Endotoxin- and D-galactosamine-induced liver injury improved by the administration of Lactobacillus, Bifidobacterium and blueberry. Dig Liver Dis 39, 849-856.

15. Osman N, Adawi D, Ahrné S, et al. (2008) Probiotics and blueberry attenuate the severity of dextran sulfate sodium (DSS)-induced colitis. Dig Dis Sci 53, 2464-2473.

16. Gram L, Ravn L, Rasch M, et al. (2002) Food spoilage - interactions between food spoilage bacteria. Int J Food Microbiol 78, 79-97.

17. Cani PD, Amar J, Iglesias MA, et al. (2007) Metabolic endotoxemia initiates obesity and insulin resistance. Diabetes 56, 1761-1772

18. Dicksved J, Halfvarson J, Rosenquist M, et al. (2008) Molecular analysis of the gut microbiota of identical twins with Crohn's disease. ISME J 2, 716-727.

19. Wang M, Molin G, Ahrné S, et al. (2007) High proportions of proinflammatory bacteria on the colonic mucosa in a young patient with ulcerative colitis as revealed by cloning and sequencing of $16 \mathrm{~S}$ rRNA genes. Dig Dis Sci 52, 620-627.

20. Wang M, Karlsson C, Olsson C, et al. (2008) Reduced diversity in the early fecal microbiota of infants with atopic eczema. J Allergy Clin Immunol 121, 129-134.

21. Karlsson C, Ahrné S, Molin G, et al. (2010) Probiotic therapy to men with incipient arteriosclerosis initiates increased 
bacterial diversity in colon: a randomized controlled trial. Atherosclerosis 208, 228-233.

22. Magurran AE (2004) An index of diversity. In Measuring Biological Diversity. Oxford: Blackwell Science Ltd.

23. Hollander M \& Wolfe DA (1999) Nonparametric Statistical Methods, 2nd ed. New York: John Wiley, Sons, Inc.

24. Hothorn T, Hornik K, van de Wiel MA, et al. (2006) A lego system for conditional inference. Am Stat 60, 257-263.

25. Trygg J (2004) Prediction and spectral profile estimation in multivariate calibration. J Chemometrics 18, 166-172.

26. Wiklund S, Johansson E, Sjöström L, et al. (2008) Visualization of GC/TOF-MS-based metabolomics data for identification of biochemically interesting compounds using OPLS class models. Anal Chem 80, 115-122.

27. Srinivasan M, Katewa SD, Palaniyappan A, et al. (2006) Maternal high-fat diet consumption results in fetal malprogramming predisposing to the onset of metabolic syndrome-like phenotype in adulthood. Am J Physiol Endocrinol Metab 291, E792-E799.

28. Bayol SA, Farrington SJ \& Stickland NC (2007) A maternal 'junk food' diet in pregnancy and lactation promotes an exacerbated taste for 'junk food' and a greater propensity for obesity in rat offspring. Br J Nutr 98, 843-851.

29. Wang M, Ahrné S, Antonsson M, et al. (2004) T-RFLP combined with principal component analysis and $16 \mathrm{~S}$ rRNA gene sequencing: an effective strategy for comparison of fecal microbiota in infants of different ages. J Microbiol Methods 59, 53-69.

30. Kadooka Y, Sato M, Imaizumi K, et al. (2010) Regulation of abdominal adiposity by probiotics (Lactobacillus gasseri SBT2055) in adults with obese tendencies in a randomized controlled trial. Eur J Clin Nutr 64, 636-643.

31. Luoto R, Kalliomäki M, Laitinen K, et al. (2010) The impact of perinatal probiotic intervention on the development of overweight and obesity: follow-up study from birth to 10 years. Int J Obes (Lond) 34, 1531-1537.

32. Fuentes S, Egert M, Jimenez-Valera M, et al. (2008) Administration of Lactobacillus casei and Lactobacillus plantarum affects the diversity of murine intestinal lactobacilli, but not the overall bacterial community structure. Res Microbiol 159, 237-243.

33. Montesi A, Garcia-Albiach R, Pozuelo MJ, et al. (2005) Molecular and microbiological analysis of caecal microbiota in rats fed with diets supplemented either with prebiotics or probiotics. Int J Food Microbiol 98, 281-289.

34. Nilsson C, Larsson BM, Jennische E, et al. (2001) Maternal endotoxemia results in obesity and insulin resistance in adult male offspring. Endocrinology 142, 2622-2630.

35. Taes YE, De Bacquer D, De Backer G, et al. (2005) Haptoglobin and body mass index. J Clin Endocrinol Metab 90, 594, author reply 594-595.

36. Perez-Echarri N, Perez-Matute P, Marcos-Gomez B, et al. (2008) Differential inflammatory status in rats susceptible or resistant to diet-induced obesity: effects of EPA ethyl ester treatment. Eur J Nutr 47, 380-386.

37. Due A, Toubro S, Stender S, et al. (2005) The effect of diets high in protein or carbohydrate on inflammatory markers in overweight subjects. Diabetes Obes Metab 7, 223-229.

38. Fantuzzi G (2005) Adipose tissue, adipokines, and inflammation. J Allergy Clin Immunol 115, 911-919.

39. Wejman JC, Hovsepian D, Wall JS, et al. (1984) Structure of haptoglobin and the haptoglobin-hemoglobin complex by electron microscopy. J Mol Biol 174, 319-341.

40. Eaton JW, Brandt P, Mahoney JR, et al. (1982) Haptoglobin a natural bacteriostat. Science 215, 691-693.

41. Smarda J, Smajs D \& Horynova S (2006) Incidence of lysogenic, colicinogenic and siderophore-producing strains among human non-pathogenic Escherichia coli. Folia Microbiol (Praba) 51, 387-391.

42. Siegmund B, Rieder F, Albrich S, et al. (2001) Adenosine kinase inhibitor GP515 improves experimental colitis in mice. J Pharmacol Exp Ther 296, 99-105.

43. Tolosano E, Fagoonee S, Hirsch E, et al. (2002) Enhanced splenomegaly and severe liver inflammation in haptoglobin/ hemopexin double-null mice after acute hemolysis. Blood 100, 4201-4208.

44. Esposito E, Iacono A, Bianco G, et al. (2009) Probiotics reduce the inflammatory response induced by a high-fat diet in the liver of young rats. J Nutr 139, 905-911.

45. Li YT, Wang L, Chen Y, et al. (2010) Effects of gut microflora on hepatic damage after acute liver injury in rats. $J$ Trauma 68, $76-83$.

46. Erridge C, Attina T, Spickett CM, et al. (2007) A high-fat meal induces low-grade endotoxemia: evidence of a novel mechanism of postprandial inflammation. Am J Clin Nutr 86 1286-1292. 